\title{
Design and Analysis of DYC and Torque Vectoring using Multiple-Frequency Control Electronic Differential in an Independent Rear Wheel Driven Electric Vehicle
}

\author{
Sujan Neroula, Santanu Sharma
}

\begin{abstract}
Electric vehicle (EV) are being embraced in recent times as they run on clean fuel, zero tail emission and are environment-friendly. Recent advancements in the field of power electronics and control strategies have made it possible to the advent in the vehicle dynamics, efficiency and range. This paper presents a design for traction control system (TCS) for longitudinal stability and Direct Yaw Control (DYC) for lateral stability simultaneous. The TCS and DYC is based on multiple frequency controlled electronic differential with a simple and effective approach. Along with it, some overviews have been presented on some state of the art in traction control system (TCS) and torque vectoring. The developed technique reduces nonlinearity, multisensory interfacing complexity and response time of the system. This torque and yaw correction strategy can be implemented alongside fuzzy control, sliding mode or neural network based controller. The effectiveness of the control method has been validated using a lightweight neighbourhood electric vehicle as a test platform. The acquired results confirm the versatility of proposed design and can be implemented in any $D C$ motor based TCS/DYC.
\end{abstract}

Keywords: Direct yaw-moment control, electric vehicle, Traction control, vehicle stability, electronic differential.

\section{INTRODUCTION}

In the current scenario, with depleting fuel sources and growing concern for the environment, Electric vehicle enjoys superiority among the other alternative fuel vehicles [1]. The sudden rise in the demand of $\mathrm{EV}$ can be attributed to its zero carbon footprint or increasing fuel price [2-4]. As other conventional vehicle must include an engine, drive train and mechanical components like differential and drive shafts, EVs have the liberty to house the traction motor in the wheel or any other configuration. This enhances occupant safety,

Revised Manuscript Received on December 30, 2019.

* Correspondence Author

Sujan Neroula, Department of Electronics and Communication Engineering, Tezpur University, Tezpur, India.

Santanu Sharma*, Department of Electronics and Communication Engineering, Tezpur University, Tezpur, India.

(c) The Authors. Published by Blue Eyes Intelligence Engineering and Sciences Publication (BEIESP). This is an open access article under the CC BY-NC-ND license (http://creativecommons.org/licenses/by-nc-nd/4.0/) efficiency, and space. Before putting forward the idea of multiple frequency controlled torque vectoring, A study on the torque vectoring and control strategies have been done with reference to the current advancements in automotive technologies and the literature.

An electric vehicle capable of direct yaw moment control with active traction control was first reported in 1996 [5]. An effective approach to traction control is by considering the stearing feedback $(\theta)$, accelerator pedal signal (APS) and brake pedal signal (BPS) [6]. However for a hierarchical control algorithm with monitoring, desired dynamics and controlled allocation of traction to wheels, it requires some additional inputs like yaw rate $(\gamma)$, slip angle $(\beta)$, forward acceleration $\left(a_{\mathrm{x}}\right)$, lateral acceleration $\left(a_{\mathrm{y}}\right)$, vehicle speed $\left(v_{\mathrm{x}}\right)$ and frictional coefficient between tyre and road $(\mu)$. The system identifies the current state of the vehicle and if any discrepancy persists, an adaptive control strategy is employed to bring the $\gamma$ and $\beta$ to the desired level. This is accomplished by bringing a moment about the $\mathrm{z}$-axis by employing asymmetric braking force or traction. The effectiveness of the control system can be evaluated by standard tests designed by International Standard (ISO3888) [6].

A typical layout of the control strategy contains

I. Control of side slip angle and yaw rate (Fuzzy, PID, Optimal LQ control and sliding mode control)

II. Allocation of torque (Traction Limitation and torque vectoring)

III. Wheel dynamics control (Wheel longitudinal slip control)

IV.Electric motor characterization (Dynamic response, efficiency, and torque-speed)

Another Stability control system is Vehicle Dynamic Control (VDC) [7]. The objective of the VDC is to deliver the vehicle response in accordance with the driver's intention. The vehicle side slip angle and yaw rate can directly influence vehicle stability [8]. During cornering the lateral acceleration increases, this consequently reduces the yaw moment generated by the stearing wheels. This was first illustrated using $\beta$-model by Shibahata, et al [9].

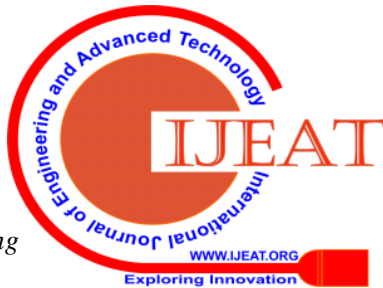


The reduced control of the stearing can bring about instability. The stability can be restored by additional external yaw moment $[10,11]$. One approach is to optimally distribute the slip angle and slip ratio to induce desired lateral and longitudinal forces $[12,13]$. The compensation of yaw moment can also be done by differential braking [14]. This system can be easily installed in existing braking systems equipped with Antilock Braking Systems (ABS) [15].

\section{A. Control Strategies.}

The key point of dynamic stability control is to find the correction required in the yaw moment. This is followed by the control strategy applied to obtain the desired yaw rate. Literature suggests various control strategies, some of them have been discussed here.

$\mathrm{Li}$, et al have proposed a fuzzy based controller to calculate the desired yaw rate by making use of instantaneous slip angle error and yaw rate error [12]. The desired yaw rate is obtained using ABS or TCS. In another reported work, Zhao, et al. have designed a sliding mode controller to provide the corrective stearing feed. In addition, an active front stearing strategy has been used for the yaw rate correction [16]. A fuzzy-based direct yaw control on an all-wheel drive (AWD) vehicle has been proposed by Tahami, et al. In their work they have reported a strategic control loop to control the yaw rate and slip angle. A reference yaw rate based on vehicle speed and stearing feedback is generated using fuzzy logic to prevent any overshooting of the corrective yaw rate [17]. Kim, at al. have reported a control strategy in which two PID control has been used for lateral acceleration error and yaw rate. Along with these, a sliding mode controller has been used for the wheel slip error. Together these signals control the motor torque used for corrective yaw rate [18] [19]. From the above studies, it can be observed that the major strategies in vehicle dynamic control involve fuzzy logic, sliding mode control and neural network.

Direct yaw control (DYC) is another very effective way to achieve the desired yaw rate in extreme situations [20], when compared to other techniques such as 4 wheel stearing [21, 22]. However, the DYC achieved by asymmetric braking has several undesirable effects. One of it is the reduction in vehicle speed that overrules the driver intention [22]. In the recent times, the active yaw control has been a topic of interest and several strategies for side-slip control, DYC and both simultaneously has been reported [21] [23-28].

Apart from these architectures, Research work can be found on model predictive control (MPC) [29]. These techniques are able to suppress the large tracking error arising due to not considering the transient behaviors during the initialization of the manoeuvre [25] [29]. The MPC has also been employed to achieve the autonomous driving mode in electric vehicles [30].

In this paper, a multi-frequency-DYC technique has been proposed for an independent rear wheel driven EV. Here a single Pulse Width Modulated (PWM) comprises of two different frequency spectrum. The duty cycle of these signals can be controlled independently. However, the duty cycle of the final signal is the function of the duty cycle of the individual frequency components.
In the framework established by the introduction, the discussions hereafter are divided into sections comprising a model to determine the necessary corrective yaw moment. Formula-based empirical model and mathematical simulations along with the characterization of the motor. The results in contexts of vehicle dynamics and DYC in real world as well in the simulation environment are discussed in the result and discussion section.

\section{THEORY}

\section{A. Vehicle stearing geometry and lateral stability.}

The Ackerman geometry, invented by Rudolf Ackerman in the 19th century. Enables the perfect rolling condition of wheels in concentric circles while negotiating a turn. Figure. 1 illustrates the toe out condition that arises due to the geometry, creating an ideal angle of the front wheels to carry out the turning manoeuvre. The inner and the outer wheel angle have been indexed as $\delta_{\mathrm{i}}$ and $\delta_{\mathrm{o}}$ respectively. Many works reported in international arenas are based on four wheel steered vehicles along with DYC [31]. In addition to this, lateral stability is a crucial factor in vehicles. Loosing of stability in dynamic condition can lead to catastrophe. One of the most important factor leading to the instability is the lowering of tire road adhesive properties [32].

For system like DYC, various system devices are involved. Therefore, challenges are encountered while applying a holistic approach. Hence a structural software and algorithms are required for a specific problem. Many key relations has to be established prior to feeding the problem to the solver. This implies to the requirement of knowledge of more physical parameters.

Depending on the direction of approach, the model can be labelled as forward and backward [33]. The forward approach model is also termed as ICE (or electric motor) to wheel model. This model is synthesized initiating from the power source and ends at wheel, considering the components like transmission and differential in between. The forward approach excels in evaluation of control algorithms. On the other hand, the backward approach first takes into account the required tractive effort at the wheels, and successively moves backwards to the power source through various powertrain components. The backward systems are mostly quasi-static model, these models give the liberty to analyze various tire forces allowing controlled power delivery from the sources [34].

Another major classification for these models are causal and non-causal: principle of cause and effect is used to describe a causal system behavior. In causal systems, the output is an indispensable function of the input parameters. This introduces a time delay in the output, gradually leading to the solution to the effect or a problem. Whereas the non-causal system deals with anticipatory problems. In these models, the cause cannot be taken into consideration and the algorithm has to take into account this anticipatory problems during operations [35]. 


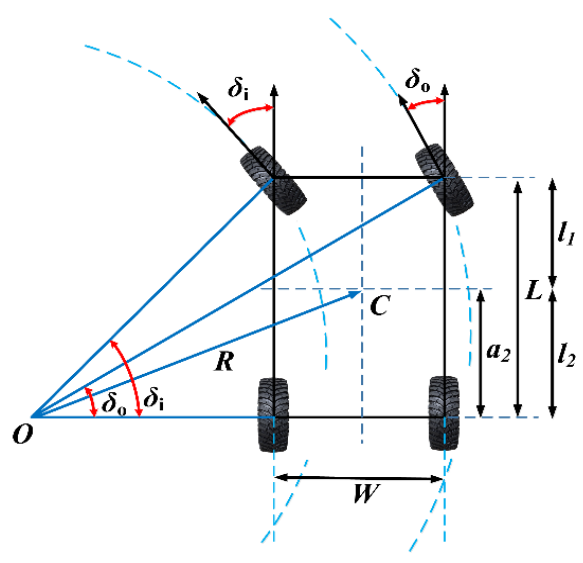

Fig. 1. Illustration of Ackerman geometry.

Here, a backward and causal approach has been taken in the designing of the multiple frequency based DYC technique. Wherein, if the acceleration is considered to be nil, the steering is considered as the primary cause that leads to change in other variables like wheel angle, turning radius, rear wheel speed, and lateral acceleration.

To have a perfect rolling contact based on the steering angle and vehicle speed, the angular speed of the inner and rear wheels must abide by the relation expressed in equation 1.

$$
\left[\begin{array}{c}
\omega_{o} \\
\omega_{i}
\end{array}\right]=\frac{v}{2 r L}\left[\begin{array}{l}
2 L+W \tan \delta \\
2 L-W \tan \delta
\end{array}\right]
$$

Dynamic and quasi-static model can better describe the vehicle dynamics, and these models can yield better accuracy and superior control. However, these models requires higher computational speed and powerful ECUs. Whereas a steady state model can make use of lookup tables for some parameters that are less prone to change in dynamic condition, alongside taking into account some real time parameters in quantized form.

At lower longitudinal velocities, the turning radius can be expressed as the function of the steering wheel alone. The radius of turn is large for a lower value of the stearing angle, therefore both the inner and outer wheels have approximately similar angle with respect to the vehicle chassis. Hence a single regression equation can define the lookup table (relation) between the two. However, beyond the $240^{\circ}$ turn of the stearing in either direction, the turning radius reduces below $10 \mathrm{~m}$. Therefore a significant difference in the two front wheel angles is observed. Therefore the relation between the stearing and wheel angle post $240^{\circ}$ of stearing turn has therefore been defined by two different regression equations as illustrated in TABLE I.

Table- II: Name of the Table that justify the values

\begin{tabular}{|c|c|c|}
\hline Stearing angle $(\boldsymbol{\theta})$ & $\delta_{i}$ & $\delta_{o}$ \\
\hline $0^{\circ}-240^{\circ}$ & $0.0437 \theta+0$ & $0.0437 \theta+0$ \\
\hline $240^{\circ}-480^{\circ}$ & $0.0698 \theta-6.29$ & $0.0346 \theta+2.18$ \\
\hline
\end{tabular}

Based on these equations, an adaptive multivariable algorithm has been designed to govern the wheel speed for various vehicle speed in inertial $x$-coordinates. The global Cartesian coordinates for the test track along with the inertial coordinates for the vehicle has been illustrated in figure 2 .

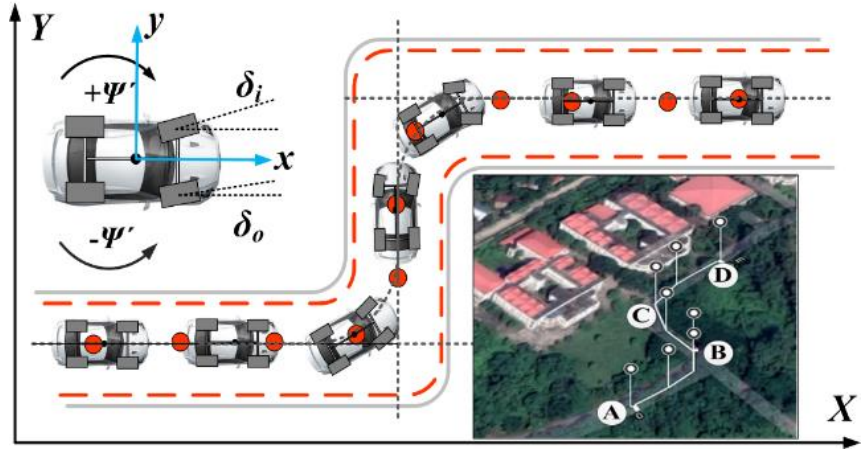

Fig. 2. The global coordinates of the test track and inertial coordinates of the vehicle.

The velocity of the vehicle in the inertial coordinates are expressed in equation 2. Here, $\Psi$ is the angle between the inertial abscissa and global abscissa. Equation 3 embodies the velocity of the vehicle in global coordinates.

$$
\begin{aligned}
& {\left[\begin{array}{c}
\dot{x} \\
\dot{y} \\
\dot{\psi}
\end{array}\right]=\left[\begin{array}{c}
\frac{r}{2}\left(\omega_{r}+\omega_{l}\right) \\
\frac{r l_{2}}{W}\left(\omega_{r}+\omega_{l}\right) \\
\frac{r}{W}\left(\omega_{r}-\omega_{l}\right)
\end{array}\right]} \\
& {\left[\begin{array}{c}
\dot{X} \\
\dot{Y} \\
\dot{\psi}
\end{array}\right]=\left[\begin{array}{c}
\frac{r}{2}\left(\omega_{r}+\omega_{l}\right) \cos \psi-\frac{l_{2} r}{W}\left(\omega_{r}-\omega_{l}\right) \sin \psi \\
\frac{r l_{2}}{W}\left(\omega_{r}+\omega_{l}\right)-\frac{l_{2} r}{W}\left(\omega_{r}-\omega_{l}\right) \cos \psi \\
\frac{r}{W}\left(\omega_{r}-\omega_{l}\right)
\end{array}\right]}
\end{aligned}
$$

The yaw moment in this case is produced by the difference in the angular velocity of the two rear wheel; so far the yaw produced by the steering has not been taken into account. The effective yaw moment produced along the vertical axis passing through the CG of the vehicle can be considered either considering the steering angle and the vehicle speed or taking into account the differential rear wheel speed and the steering. The vehicle speed in the global coordinates, taking into account the steering is defined as (equation 4)

$$
\left[\begin{array}{c}
\dot{X} \\
\dot{Y} \\
\dot{\psi}
\end{array}\right]=\frac{r\left(\omega_{r}+\omega_{l}\right)}{2}\left[\begin{array}{c}
\cos \psi \\
\sin \psi \\
\frac{\tan \delta}{L}
\end{array}\right]
$$

The effective yaw moment for the vehicle during a turn can be expressed as.

$\dot{\psi}=\frac{r\left(\omega_{r}+\omega_{l}\right) \tan \delta}{2 L}, \frac{r}{W}\left(\omega_{r}-\omega_{l}\right)$

When the vehicle wheel are made to turn merely to abide by the Ackerman geometry to achieve rolling contact with road surface while compromising a turn. Both part of equation 5 yields a moment that resembles the yaw moment expressed in equation 3 . 


\section{Design and analysis of DYC and torque vectoring using multiple-frequency control electronic differential in an independent rear wheel driven electric vehicle}

Taking into account the fact that the driver intends to carry out a sharp turn, especially at a high speed, for collision avoidance. The vehicle must generate the necessary yaw response to meet the driver's intension. In case, the steering fails to generate sufficient yaw, the DYC must assist the vehicle in such unfavorable scenarios.

\section{B. Lateral force and slip.}

Previous studies suggest that the effect on side slip angle and yaw rate are always in opposition to each other whenever a control input is applied, which can deteriorate the side slip performance for large steering angle [33].

The well-known approach to lateral stability is by curve fitting that establishes a relation called magic formula [37], magic formula leads to a relation between tyre stiffness, shape parameters, slip, curvature parameters and force.

$F_{y}=D \sin (C \arctan (B . s-E(B . s-\arctan (B . s))))(6)$

Here B, C, D and E are the constants obtained experimentally. The magic formula is further simplified as

$$
F(s)=F_{\max } \sin \left(B\left(1-e^{-|s| / A}\right) \operatorname{sign}(s)\right)
$$

Where,

$$
\begin{gathered}
B=\pi-\arcsin \left(F_{\infty} / F_{\text {max }}\right) \\
A=F_{\text {max }} B / \arctan (C)
\end{gathered}
$$

Equation defining the longitudinal slip

$$
s_{x}=f\left(F_{x}, F_{z}, \mu\right)= \begin{cases}=\frac{F_{x}}{C_{x}} ; & \text { for }\left|F_{x}\right| \leq \frac{\mu F_{z}}{2} \\ =\frac{\mu F_{z}}{4 C_{x}} \frac{\operatorname{sign}\left(F_{x}\right)}{1-\frac{\left|F_{x}\right|}{\mu F_{z}}} ; & \text { for }\left|F_{x}\right|<0.95 \mu F_{z} \\ =\frac{\mu F_{z}}{4 C_{x}} \frac{\operatorname{sign}\left(F_{x}\right)}{1-0.95} ; & \text { else }\end{cases}
$$

The longitudinal force and the lateral force can be expressed as

$$
\left[\begin{array}{c}
F_{x} \\
F_{y}
\end{array}\right]=\left[\begin{array}{c}
r \omega-v_{x} \\
-v_{y}
\end{array}\right] \frac{F_{x y}}{\sqrt{\left(r \omega-v_{x}\right)^{2}+\left(v_{y}\right)^{2}}}
$$

The wheel resulting wheel slip can be expressed as resultant of longitudinal and lateral slip

$$
\begin{aligned}
& s^{2}=s_{x}{ }^{2}+s_{y}{ }^{2}, \\
& s_{x}=\frac{r \omega-v_{x}}{r \omega} \text { And } \\
& s_{y}=\frac{v_{y}}{r \omega}
\end{aligned}
$$

These may result into neutral steer, understeer or oversteer during cornering. As illustrated in figure 4(c)

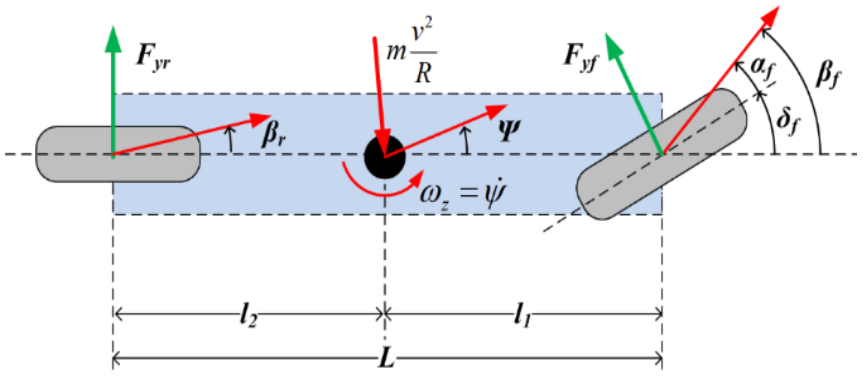

Fig. 3. Physical model illustrating side slip and turning radius.

$m \frac{v^{2}}{R} \approx F_{y r}+F_{y f} F_{y f}=-C_{f} s_{y f} F_{y r}=-C_{r} s_{y r}$

The relation between steering angle, longitudinal velocity and turning radius can be expressed as

$\delta_{f}=\frac{C_{f} C_{r} L^{2}+\left(C_{r} l_{2}+C_{r} l_{1}\right) m v_{x}^{2}}{C_{f} C_{r} L+\left(C_{r} l_{1}+C_{r} l_{2}\right)} \frac{\omega_{z}}{v_{x}}$

$\omega_{z}=\frac{C_{f} C_{r} L+\left(C_{r} l_{1}+C_{r} l_{2}\right) F_{f x}}{C_{f} C_{r} L^{2}+\left(C_{r} l_{2}-C_{f} l_{1}\right) m v_{x}^{2}} v_{x} \delta_{f}$

$v_{y}=\frac{C_{f}\left(C_{r} L l_{2}-l_{1} m v_{x}^{2}\right)+\left(C_{r} L l_{2}-l_{1} m v_{x}^{2}\right) F_{f x}}{C_{f} C_{r} L^{2}+\left(C_{r} l_{2}-C_{f} l_{1}\right) m v_{x}^{2}} v_{x} \delta_{f}$

(15)

The relation between yaw angle, turning radius and longitudinal velocity can be expressed as.

$$
\beta=\tan ^{-1}\left(\frac{1}{R}\left(l_{2}-\frac{l_{1} m v_{x}^{2}}{L C_{r}}\right)\right)
$$

The normalised required steering angle is expressed as

$$
\frac{\delta_{f} R}{L}=1+\chi_{u} \frac{m v_{x}^{2}}{L}
$$

Here $\chi_{\mathrm{u}}$ is the understeer gradient

$$
\chi_{u}=\frac{C_{r} l_{2}-C_{f} l_{1}}{C_{f} C_{r} L}
$$

Which is obtained to be

$3.73 \times 10^{-6} \mathrm{~N}^{-1}$, for understeer

$0 \times 10^{-6} \mathrm{~N}^{-1}$ for neutral steer and

$-3.96 \times 10^{-6} \mathrm{~N}^{-1}$ over steer conditions

For a vehicle the critical speed is the speed at which the vehicle becomes unstable and a significant growth in yaw moment is observed for even a small disturbances in input parameters.

Our results suggest that a zero steering angle is required for the oversteered vehicle at critical speed of $36 \mathrm{~m} / \mathrm{s}$. another important aspect of steering dynamics is the vehicle characteristic speed, the speed at which the vehicle requires twice the steering angle than required to manoeuvre on the same track in lower speed. The characteristic speed obtained from the simulation results for the designed vehicle in the given parameters is found to be $33 \mathrm{~m} / \mathrm{s}$ as illustrated in figure 4(b). 

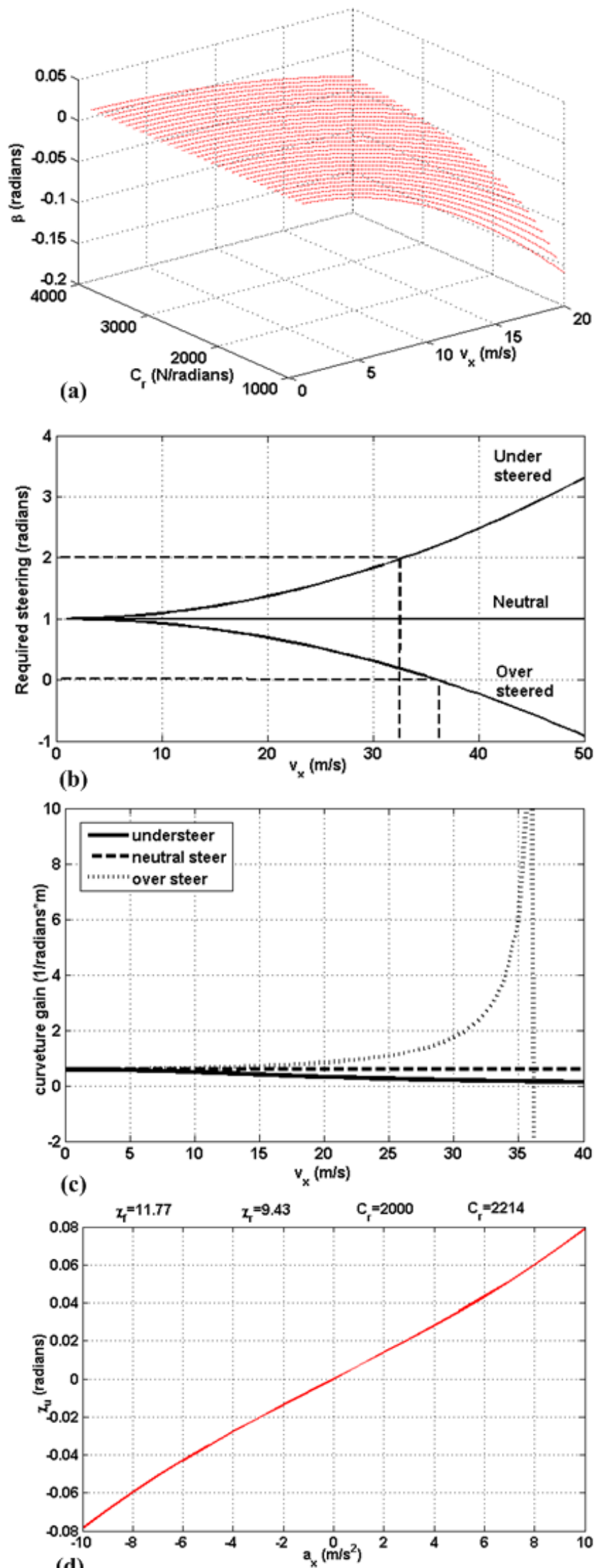

(d)

Fig. 4. (a) Yaw vs $C_{\mathrm{r}}$ for various longitudinal velocities. (b) Stearing angle for steady state cornering. (c) Curvature gain in neutral steer, under steer and over steer conditions in steady state cornering. (d) Variation of Understeer gradient for different longitudinal acceleration.

The understeer gradient is also largely influenced by the

vehicle longitudinal acceleration. The relation between these is established in equation 19.

$$
\begin{gathered}
\chi_{u}=\frac{m g l_{2}}{\frac{C_{f}}{F_{z f}}\left(m g \frac{l_{2}}{L}-m a_{x} \frac{h}{L}\right) L}-\frac{m g l_{1}}{\frac{C_{r}}{F_{z r}}\left(m g \frac{l_{2}}{L}+m a_{x} \frac{h}{L}\right)} \\
\chi_{f}=\frac{C_{f}}{F_{z f}}=2214 / 188=11.77 \\
\chi_{r}=\frac{C_{r}}{F_{z r}}=2000 / 212=9.43
\end{gathered}
$$

The vehicle wheel speed has to regulate such that, a yaw moment is created by the differential wheel speed. The differential speed higher than the necessary wheel speed to abide by the Ackerman geometry will bring about an additional curvature gain. This will the counter the lateral acceleration and any undesired understeer condition arising due to it. Following sections focuses on the design of the multiple frequency electronic differential for control of lateral and longitudinal acceleration.

\section{MULTIPLE FREQUENCY CONTROLLED DYC}

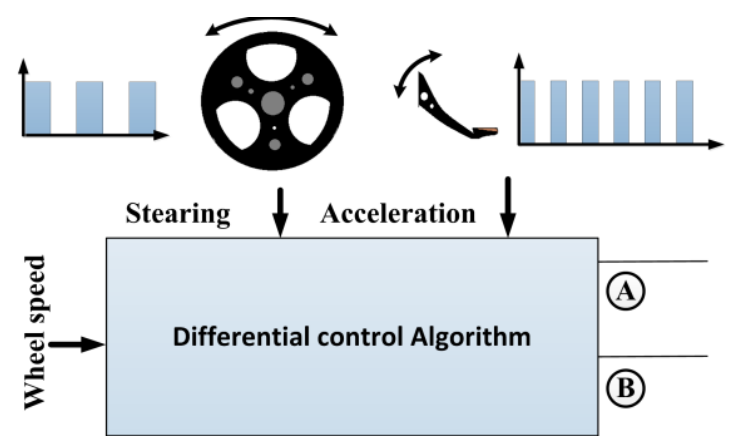

Fig. 5. Multiple frequency control differential algorithm.

In order to avoid complications, a single quadrant chopper with power MOSFETs has been designed as a low side chopper drive. The schematics of the drive circuit is illustrated in Fig. 6, labeled as a motor drive, chopper and snubber. The drive motor of a vehicle compromising a turn depends on various parameters such as vehicle speed, turning radius, stearing angle, and lateral slip. To carry out a smooth turning manoeuvre, the control variable chosen in this work are stearing angle and accelerator position. The accelerator pedal controls the duty cycle $(D)$ of the higher frequency PWM signal, indexed as signal A. Linear relation exists between the accelerator pedal position and $D$. On the contrary, the duty cycle $(d)$ of the lower frequency signal is a function of the stearing angle.

The relation between $d$ and $\theta$ is established using the correlation equations derived as illustrated in Table I. The duty cycle of the final signal is a function of $d$ and $D$. while executing a left turn, the duty cycle of signal $C$ decreases consequently the duty cycle of signal $D$ increases.

This difference in the torque generates a torque about the vehicle z-axis about the CG of the vehicle

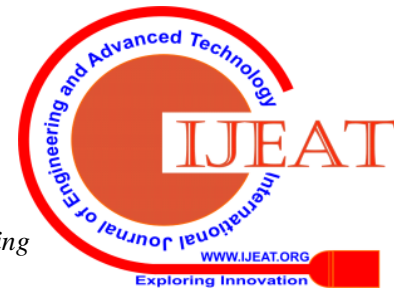




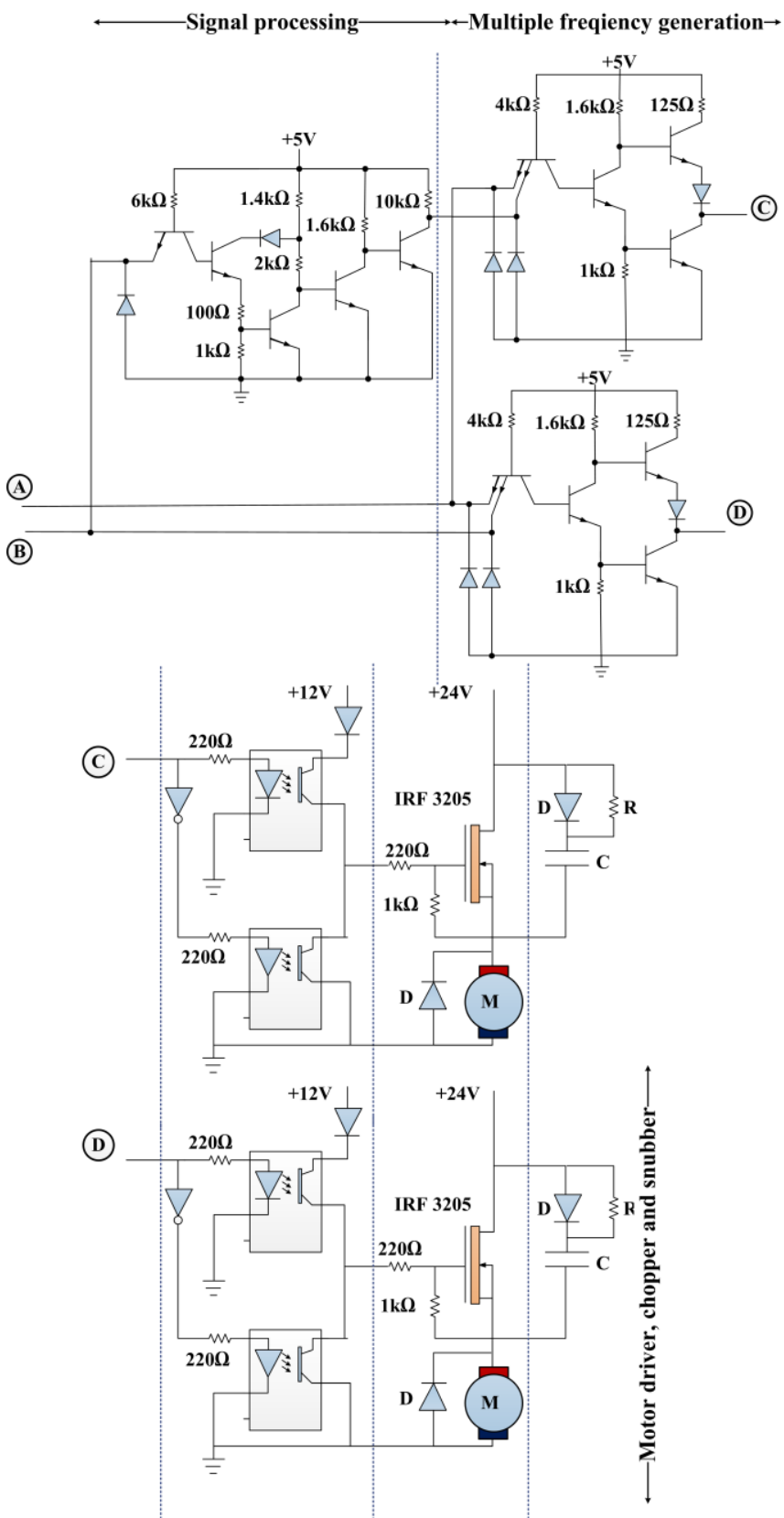

Fig. 6. Circuit to realize the multiple frequency controlled electronic differential.

The additional torque generated due to the difference in the speed of two rear wheel helps the vehicle to overcome any understeer situation. The torque can be expressed as.

$$
\tau_{z}=\frac{W}{2} \frac{C_{r} r}{v_{x}}\left(\omega_{l}-\omega_{r}\right)
$$

\section{RESULTS AND DISCUSSION}

Optimal control strategy for slip-tracking drastically reduces the tire slip trends and distributes the required torque abiding by the control-allocation algorithm following the reference slip value [38].

To illustrate the effectiveness and validity of the proposed DYC technique on an electric vehicle, numerous tests were carried out. The tests were conducted on a test track using a lightweight NEV.
The NEV was designed with two independent motor and equipped with various sensors to keep track of speed, stearing angle, motor current, motor voltage, wheel load, lateral acceleration and longitudinal acceleration. The drivetrain of the vehicle has been designed with a two-stage chain drive having a reduction ratio of 10:1 .The experiment was designed to carry out a drive cycle in which the vehicle has to undergo a left turn of radius $35 \mathrm{~m}$. Followed by a right turn of radius $38 \mathrm{~m}$. The initial and the final point has been indexed as $\mathrm{A}$ and $\mathrm{D}$ in the map.

The motor speed was strictly regulated in accordance with the above equations. This helps the vehicle to abide the Ackerman stearing geometry during neutral steer condition, and to achieve required curvature gain in situation of understeer.

The test was carried out on the test track at a uniform speed of $10 \mathrm{~m} / \mathrm{s}$. The real-time result obtained for the drive cycle has been presented in Fig. 9 and 10.

The stearing angle of the vehicle has been recorded in real time, the initial point and final point has been indexed as $\mathrm{A}$ and $\mathrm{D}$. the maximum required stearing angle for the maneuvering of the vehicle at point B and C is $+214^{\circ}$ and $-211^{\circ}$. Monitoring the steering angle has been done using optical rotary encored with accuracy of $0.225^{\circ}$. The corresponding turning radius was recorded by the on-board accelerometers and vehicle speed sensor. The turning radius of the vehicle in straight path keeps varying between negative and positive infinity. However a finite value is recorded when the vehicle enters a turn. In addition, the corresponding lateral and longitudinal acceleration has been recorded. These data are vital for the implementation of the DYC technique using the multiple frequency controlled electronic differential. The frequency associated with the steering and accelerator along with the final frequency to traction motors has been illustrated in figure 7 .

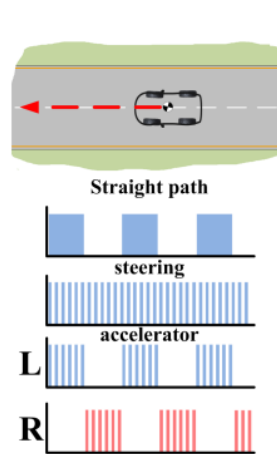

(a)

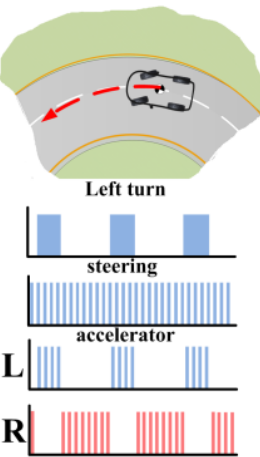

(b)

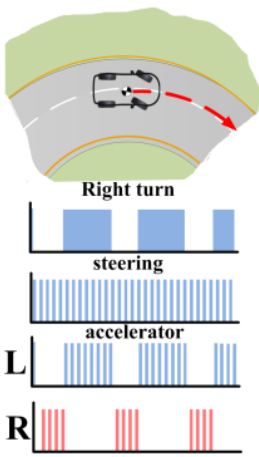

(c)
Fig. 7. Illustration of multiple frequency signals for (a) vehicle on a straight path. (b) vehicle taking a left turn. (c) vehicle taking a right turn.

Two different frequency signals has been employed to realize the electronic differential design. The first signal with higher frequency has the information about the vehicle accelerator position. This signal has been superimposed upon the second signal of lower frequency with information regarding the vehicle stearing angle. 
Together, these signals are used to control the speed and torque of the inner and outer traction motors. The real time steering angle along with the recorded turning radius has been embodied in figure 8(a) and (b) respectively.
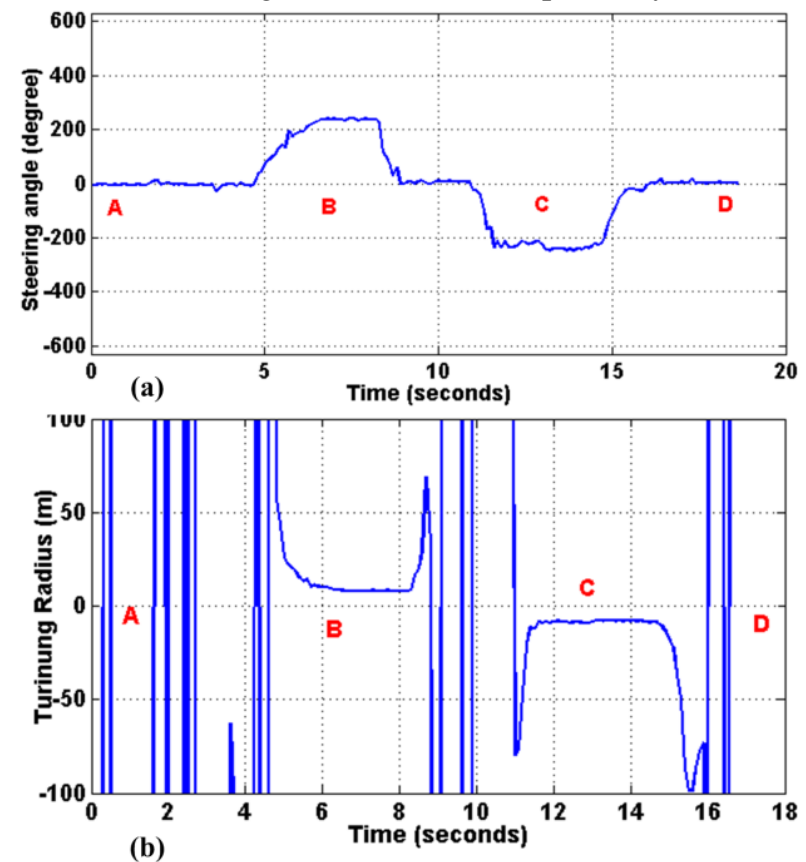

Fig. 8. (a) Required steering angle on the track (speed= $10 \mathrm{~m} / \mathrm{s})$. (b) turning radius recorded in real time (speed $=10 \mathrm{~m} / \mathrm{s}$ )

The duty cycle of the higher frequency signal controls the longitudinal acceleration of the vehicle. Whereas the duty cycle of the lower frequency signal is used to implement the electronic differential during the turning manoeuvre. This differential design is also capable of implementing the necessary yaw correction strategy in situation of under-steer and over-steer.

The figure 9 shows the recorded stearing angle, normalized front wheel angle and the real-time yaw angle for the steering angle at longitudinal speed of $10 \mathrm{~m} / \mathrm{s}$. the yaw angle has been recorded for two situations; firstly the vehicle steering was aligned to carry out neutral steering and second yaw moment was recorded with the tuning of steering for over-steer. In the following part, the objective would be to recreate similar results with neutral steering and implementing the DYC technique.

With the DYC on, and steering tuned for neutral steer, the steering angle, current associated with the left and right wheel and the duty cycle of the lower frequency signal has been recorded in real time. This illustrates a significant rise in the current of the outer motor and a dip in the value of the recorded current

to the inner wheel during a turning maneuver. A variation in the duty cycle of one signal is brought without any disturbances to the second signal. This ensures no variation in the vehicle longitudinal acceleration or instantaneous speed of the vehicle as the DYC carries out the yaw correction
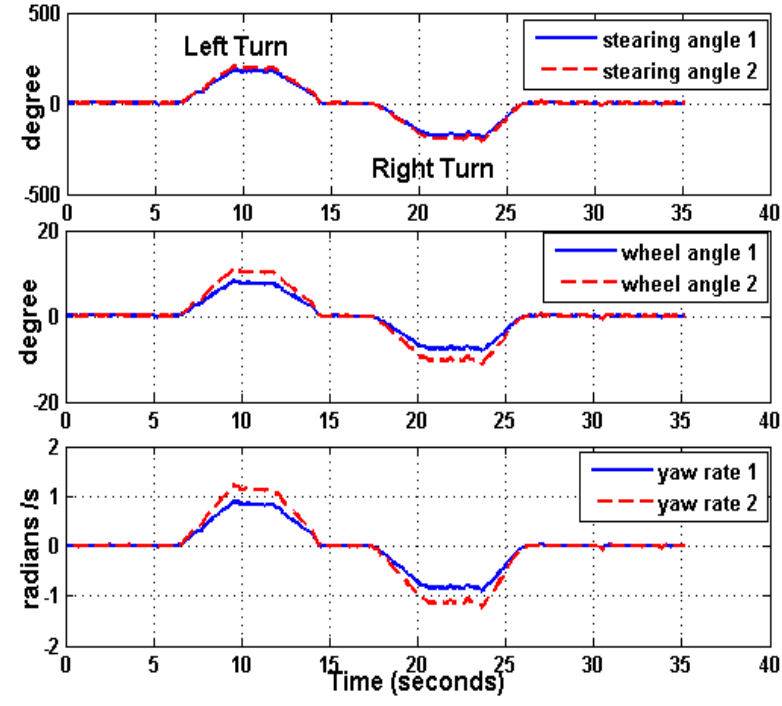

Fig. 9. Steating angle, wheel angle and yaw moment for two diffeerent tests $($ speed $=10 \mathrm{~m} / \mathrm{s})$.
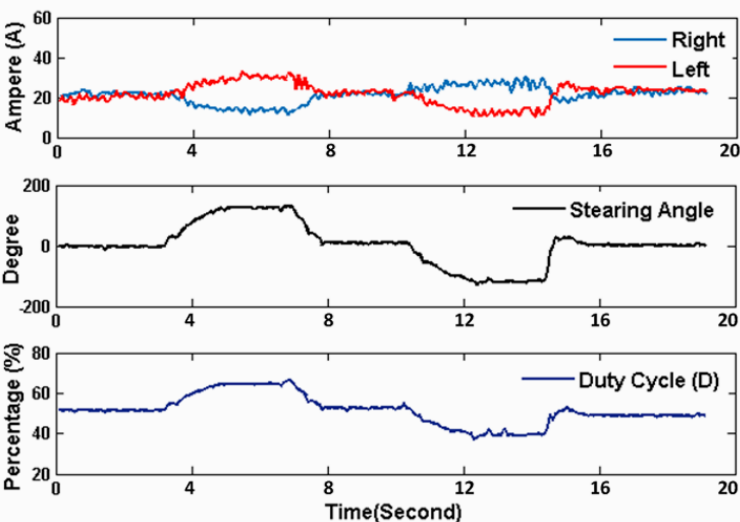

Fig. 10. Real time representation of current drawn by the left and right traction motors, steering angle and duty cycle of the frequency associated to steering.

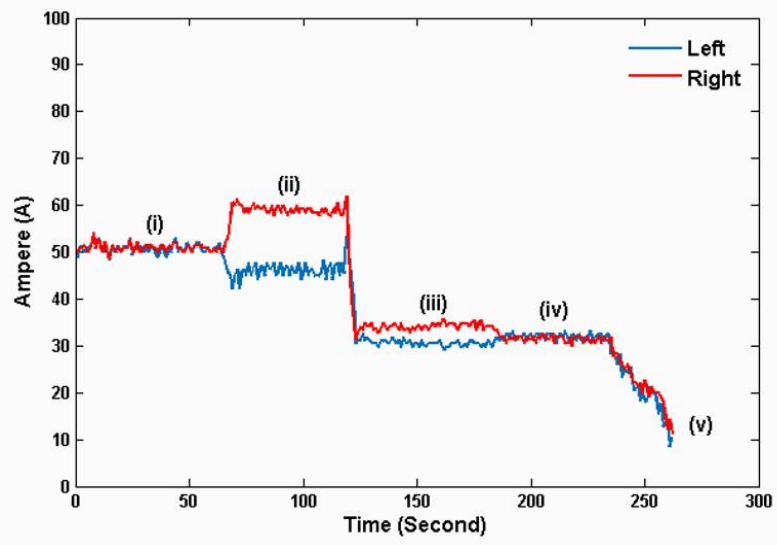

Fig. 11. Real time plot to illustrate the behaviour of the differential for deceleration during turning manoeuvre

Along with these, it was important to observe the behaviour of the design when both the control variable changed simultaneously. The uniqueness of the solution to address the problem of traction control and DYC is being the ability of two input parameters to control the PWM duty cycle independently.

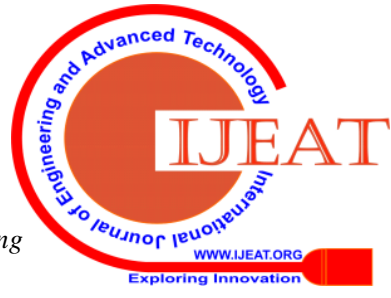




\section{Design and analysis of DYC and torque vectoring using multiple-frequency control electronic differential in an independent rear wheel driven electric vehicle}

Even though the frequency of each input signal is unique, situations may occur when simultaneous changing of both parameters can compromise the vehicle stability. Therefore high speed tests were carried out to rule out these speculation, this test was accomplished by sudden decelerating the vehicle during a turn. Figure 11, shows the current drawn by the motor when the vehicle decelerates during cornering. The region of the plots are divided into five sections indexed as (i) to (v), (i): the vehicle is driven on a straight path at uniform velocity, (ii): the vehicle enters a sharp turn, hence a significant change in the two current values can be observed, (iii): the vehicle undergoes rapid deceleration while negotiating the turn, (iv): the vehicle completes the turn. This test illustrates the ability of the system to handle the situation which might otherwise bring about instability in the vehicle.

\section{CONCLUSIONS}

This paper presents the traction control and DYC for electric vehicle with two independently driven rear wheels, based on multiple frequency electronic differential the results were compared with conventional traction control strategies. The novel conclusion of the paper are as follows

1) The multiple frequency controlled electronic differential presents a simple though effective approach to carry out a DYC action in an independent rear wheel driven electric vehicle. Analysis, simulation, and tests confirm the effectiveness of the proposed electronic differential design.

2) From the results, it can be affirmed that this design will enhance the vehicle efficiency and the range to some extent. Moreover, this simple approach can considerably reduce the design complexity and manufacturing cost of power electronics essential for utility electric vehicles. Multiple driving cycles affirm the effectiveness of the DYC using the multiple frequency control technique. Moreover, hardware realization of a single PWM duty cycle as a function of multiple parameters without complications and requirement of complex algorithm is first of its kind.

3) The yaw control strategy presented here exhibits superior responsiveness and no lag is observed between the control variables and differential action. Moreover the multiple frequency control of DC motor speed can be designed by incorporating numerous frequency signals, with each frequency having a distinct characteristics of one or more control variable.

Future developments of the research will include.

1) Increase in the number of variables and vehicle parameters for nonlinear predictive control strategies.

2) Inclusion of passenger occupancy and taking into account the variation in vehicle centre of gravity with different passenger occupancy.

\section{REFERENCES}

1. R. Rüther, L. C. P. Junior, A. H. Bittencourt, L. Drude, and I. P. D. Santos, "Strategies for Plug-in Electric Vehicle-to-Grid (V2G) and Photovoltaics (PV) for Peak Demand Reduction in Urban Regions in a Smart Grid Environment," Plug In Electric Vehicles in Smart Grids Power Systems, pp. 179-219, 2014.

2. S. Ji, C. R. Cherry, M. J. Bechle, Y. Wu, and J. D. Marshall, "Electric Vehicles in China: Emissions and Health Impacts," Environmental Science \& Technology, vol. 46, no. 4, pp. 2018-2024, 2012.

3. Z. Rezvani, J. Jansson, and J. Bodin, “Advances in consumer electric vehicle adoption research: A review and research agenda," Transportation Research Part D: Transport and Environment, vol. 34, pp. 122-136, 2015

4. J. Wideberg, C. Bordons, P. Luque, D. A. Mántaras, D. Marcos, and H. Kanchwala, "Development and Experimental Validation of a Dynamic Model for Electric Vehicle with in Hub Motors," Procedia Social and Behavioral Sciences, vol. 160, pp. 84-91, 2014.

5. K. Sawase, "Application of active yaw control to vehicle dynamics by utilizing driving/breaking force," JSAE Review, vol. 20, no. 2, pp. 289-295, 1999.

6. H. Kanchwala, J. Wideberg, C. B. Alba, and D. Marcos, "Control of an independent 4WD electric vehicle by DYC method," International Journal of Vehicle Systems Modelling and Testing, vol. 10, no. 2, p. 168, 2015.

7. J. Wang and R. Longoria, "Coordinated vehicle dynamics control with control distribution,” 2006 American Control Conference, 2006.

8. W. Cho, J. Yoon, J. Kim, J. Hur, and K. Yi, “An investigation into unified chassis control scheme for optimised vehicle stability and manoeuvrability," Vehicle System Dynamics, vol. 46, no. sup1, pp. 87-105, 2008.

9. Y. Shibahata, K. Shimada, and T. Tomari, "Improvement of Vehicle Maneuverability by Direct Yaw Moment Control," Vehicle System Dynamics, vol. 22, no. 5-6, pp. 465-481, 1993.

10.J. Fredriksson, J. Andreasson, and L. Laine, "Wheel force distribution for improved handling in a hybrid electric vehicle using nonlinear control," 2004 43rd IEEE Conference on Decision and Control (CDC) (IEEE Cat. No.04CH37601), 2004.

11.W. Cho, J. Yoon, S. Yim, B. Koo, and K. Yi, "Estimation of Tire Forces for Application to Vehicle Stability Control," IEEE Transactions on Vehicular Technology, vol. 59, no. 2, pp. 638-649, 2010.

12.L. Feiqiang, W. Jun, and L. Zhaodu, "On the vehicle stability control for electric vehicle based on control allocation," 2008 IEEE Vehicle Power and Propulsion Conference, 2008.

13.L. Feiqiang, W. Jun, and L. Zhaodu, "Motor torque based vehicle stability control for four-wheel-drive electric vehicle," 2009 IEEE Vehicle Power and Propulsion Conference, 2009.

14.A. T. V. Zanten, "Bosch ESP Systems: 5 Years of Experience," SAE Technical Paper Series, 2000.

15.A. T. V. Zanten, R. Erhardt, K. Landesfeind, and G. Pfaff, "VDC Systems Development and Perspective," SAE Technical Paper Series, 1998.

16.Y. Zhao, Y. Zhang, and Y. Zhao, "Stability Control System for Four-in-Wheel-Motor Drive Electric Vehicle," 2009 Sixth International Conference on Fuzzy Systems and Knowledge Discovery, 2009.

17.F. Tahami, R. Kazemi, and S. Farhanghi, “A novel driver assist stability system for all-wheel-drive electric vehicles," IEEE Transactions on Vehicular Technology, vol. 52, no. 3, pp. 683-692, 2003.

18.D. Kim and H. Kim, "Vehicle Stability Control with Regenerative Braking and Electronic Brake Force Distribution for a Four-Wheel Drive Hybrid Electric Vehicle," Proceedings of the Institution of Mechanical Engineers, Part D: Journal of Automobile Engineering, vol. 220, no. 6, pp. 683-693, 2006.

19.J. Kim and H. Kim, "Electric Vehicle Yaw Rate Control using Independent In-Wheel Motor,” 2007 Power Conversion Conference Nagoya, 2007.

20.M. Mirzaei, "A new strategy for minimum usage of external yaw moment in vehicle dynamic control system," Transportation Research Part C: Emerging Technologies, vol. 18, no. 2, pp. 213-224, 2010.

21.M. Abe, "Vehicle dynamics and control for improving handling and active safety: From four-wheel steering to direct yaw moment control," Proceedings of the Institution of Mechanical Engineers, Part K: Journal of Multi-body Dynamics, vol. 213, no. 2, pp. 87-101, 1999.

22.M. Selby, W. J. Manning, M. D. Brown, and D. A. Crolla, "A Coordination Approach for DYC and Active Front Steering," SAE Technical Paper Series, 2001.

23.E. Esmailzadeh, A. Goodarzi, and G. Vossoughi, "Optimal yaw moment control law for improved vehicle handling," Mechatronics, vol. 13, no. 7, pp. 659-675, 2003. 
24.O. Mokhiamar and M. Abe, "Effects of model response on model following type of combined lateral force and yaw moment control performance for active vehicle handling safety," JSAE Review, vol. 23, no. 4, pp. 473-480, 2002.

25.O. Mokhiamar and M. Abe, "How the four wheels should share forces in an optimum cooperative chassis control," Control Engineering Practice, vol. 14, no. 3, pp. 295-304, 2006.

26.M. Abe, "Side-slip control to stabilize vehicle lateral motion by direct yaw moment," JSAE Review, vol. 22, no. 4, pp. 413-419, 2001.

27.K. Park, "Controller design for improving lateral vehicle dynamic stability,” JSAE Review, vol. 22, no. 4, pp. 481-486, 2001.

28.Y. A. Ghoneim, W. C. Lin, D. M. Sidlosky, H. H. Chen, Y.-K. Chin, and M. J. Tedrake, "Integrated chassis control system to enhance vehicle stability," International Journal of Vehicle Design, vol. 23, no. 1/2, p. 124, 2000.

29.H. Kanchwala and C. Bordons, "Improving Handling Performance of an Electric Vehicle Using Model Predictive Control," SAE Technical Paper Series, 2015.

30.H. Kanchwala and H. Ogai, "Development of an Intelligent Transport System for EV," SAE International Journal of Passenger Cars Electronic and Electrical Systems, vol. 9, no. 1, pp. 9-21, 2016.

31.P. Hang, X. Chen, and F. Luo, "LPV/Hœ Controller Design for Path Tracking of Autonomous Ground Vehicles Through Four-Wheel Steering and Direct Yaw-Moment Control," International Journal of Automotive Technology, vol. 20, no. 4, pp. 679-691, Sep. 2019.

32.G. Huang, X. Yuan, K. Shi, and X. Wu, “A BP-PID controller-based multi-model control system for lateral stability of distributed drive electric vehicle," Journal of the Franklin Institute, vol. 356, no. 13, pp. 7290-7311, 2019.

33.J. Scordia, M. D. Renaudin, R. Trigui, B. Jeanneret, F. Badin, and C. Plasse, "Global optimisation of energy management laws in hybrid vehicles using dynamic programming," International Journal of Vehicle Design, vol. 39, no. 4, p. 349, 2005.

34. C. Chan, A. Bouscayrol, and K. Chen, "Electric, Hybrid, and Fuel-Cell Vehicles: Architectures and Modeling," IEEE Transactions on Vehicular Technology, vol. 59, no. 2, pp. 589-598, 2010.

35.M. Dempsey, "Dymola for Multi-Engineering Modelling and Simulation," 2006 IEEE Vehicle Power and Propulsion Conference, 2006.

36.S. Wilkins and M. Lampérth, "The Development of an Object-Oriented Tool for the Modeling and Simulation of Hybrid Powertrains for Vehicular Applications," SAE Technical Paper Series, 2003.

37. A. Shimura and K. Yoshida, "Steering Control for Car Cornering by Means of Learning Using Neural Network and Genetic Algorithm," IFAC Proceedings Volumes, vol. 31, no. 2, pp. 25-28, 1998.

38.K. Bayar, "Performance comparison of electric-vehicle drivetrain architectures from a vehicle dynamics perspective," Proceedings of the Institution of Mechanical Engineers, Part D: Journal of Automobile Engineering, p. 095440701986749, 2019.

\section{AUTHORS PROFILE}

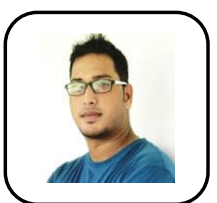

Sujan Neroula received his B-Tech degree in 2012 and has done his M-tech in Electronics Design and Technology, from Tezpur University in the year 2016, currently he is doing Ph.D. in department of Electronics and Communication Engineering, Tezpur University since 2016. His area of interests include Electric vehicle, Power Electronics, vehicle dynamics, and semiconductor devices.

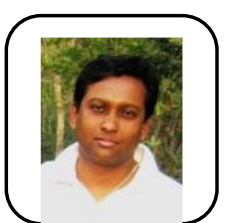

Santanu Sharma received the B.E degree in Electrica Engineering from Jorhat Engineering College, Jorhat, Assam, India (Dibrugarh University) in 1998 And M.-Tech degree in Electronics design and technology from Tezpur University, Tezpur, India, in 2000 and the Ph.D. degree in Electronics and Communication engineering from Tezpur University, in 2010.

He was a project staff in International Centre for Radio Science, Jodhpur, from July 2000 to December 2000.He was also working as Engineering Assistant at Prasar Bharati, Broadcasting Corporation of India, AIR, from December 2000 to November 2001. From December 2001 to January 2010 he was an Assistant Professor with the Electronics and Communication Engineering Department, Tezpur University, Tezpur. Currently, he is working as an Associate Professor in the same department. His area of research includes semiconductor devices, bioelectronics devices, electric vehicle and power electronics. 